\title{
Sensory Transduction in Halobacterium halobium: Retinal Protein Pigment Controls UV-Induced Behavioral Response
}

\author{
Norbert A. Dencher \\ Dept. of Biophysical Chemistry, Biocenter, University of Basel, CH-4056 Basel \\ and \\ Eilo Hildebrand \\ Institut für Neurobiologie, Kernforschungsanlage, D-5170 Jülich \\ Z. Naturforsch. 34 c, 841 - 847 (1979); received June 5/July 6, 1979 \\ Bacteria, Photophobic Response, Bacteriorhodopsin, Carotenoids, Accessory Pigment \\ Both photosystems, PS 370 and PS 565, controlling behavioral responses in Halobacterium halo- \\ bium [E. Hildebrand and N. Dencher, Nature 257, $46-48$ (1975)] are reversibly inhibited when \\ bacteria are grown in the presence of $1 \mathrm{~mm}$ nicotine which is known to block biosynthesis of reti- \\ nal. \\ Photobehavior can be restored within some minutes to hours by adding retinal to nicotine- \\ treated bacteria, PS 370 thereby reappearing earlier than PS 565. The reconstitution rate depends \\ on the concentration and on the kind of retinal isomers applied. All-trans retinal is most effective. \\ PS 370 becomes fully sensitive if reconstituted in the presence of nicotine. This rules out the possi- \\ bility that the alkaloid may directly inhibit steps of signal transmission following photoreception. \\ The action spectrum of PS 370 regenerated with retinal alone of $H$. h., strain $\mathrm{R}_{1} \mathrm{~L}_{3}$ (a mutant \\ deficient in carotenoids), fails to show all secondary peaks around $450 \mathrm{~nm}$ which in strain $R_{1}$ occur \\ besides the prominent maximum at $370 \mathrm{~nm}$. Addition of carotenoids (mainly $\alpha$-bacterioruberin) \\ to reconstituted cells of $\mathrm{R}_{1} \mathrm{~L}_{3}$ restores the sensitivity in that spectral region. Carotenoids or flavin \\ solely added to nicotine-treated bacteria cannot restore photobehavior. \\ We conclude that the active pigment of PS 370, which mediates the photophobic response to \\ increase of light intensity (step-up response), represents a retinal protein complex and that carote- \\ noids participate in photoreceptor function as accessory pigments. The biochemical relation of the \\ UV-absorbing retinal protein complex to bacteriorhodopsin is discussed.
}

Halobacteria became interesting after the role of bacteriorhodopsin (BR) in light energy conversion had been discovered by Oesterhelt and Stoeckenius $[1,2]$. It turned out that they are suitable objects also for studying photosensory processes in bacteria. A characteristic behavioral response of Halobacterium is controlled by two photosystems (PS) which are different with respect to spectral sensitivity, adaptation and other important features [3]. Halobacteria are bipolarly flagellated and swim equally well in both directions parallel to their long axis. Temporal changes of incident light trigger a sudden reversal of swimming direction which, from time to time, also occurs spontaneously. Decrease of yellow-green light elicits the "step-down photophobic response" (for terminology see ref. [4]) which is mediated by PS 565, whereas increasing light intensity in the ul-

Abbreviations: BR, bacteriorhodopsin; PS, photosystem; UV, ultraviolet; BO, bacterio-opsin; H. h., Halobacterium halobium; PM, purple membrane.

Reprint requests to N. A. Dencher.

0341-0382/79/0900-0841 \$01.00/0 traviolet (UV) and blue triggers the "step-up photophobic response" through PS 370.

Several lines of evidences led to the assumption that the active pigment of PS 565 is BR [3], a chromoprotein which contains retinal (vitamin A aldehyd) covalently linked to a lysine residue of a protein, bacterio-opsin (BO) [1]. The nature, however, of the UV-absorbing pigment of PS 370 was not known up to now. A flavin protein together with carotenoids, the latter acting as shielding pigments, has been considered as well as a retinylidene protein [3]. The experiments reported here show that most likely the latter is true and that carotenoids act mainly as accessory pigments thereby participating in the quantum catch of PS 370 .

\section{Materials and Methods}

\section{Bacteria}

Halobacterium halobium $\left(H . h\right.$.), strains $\mathrm{R}_{1}$ and $\mathrm{R}_{1} \mathrm{~L}_{3}$, were grown in sterile culture medium [5] semiaerobically at $40{ }^{\circ} \mathrm{C}$ under illumination with white light of $1 \mathrm{~mW} \mathrm{~cm}^{-2}$ as described elsewhere 
[6]. Bacteria of the stationary growth phase (about $10^{9}$ cells $/ \mathrm{ml}$ ) were used for experiments.

Retinal-free cells were obtained by culturing bacteria in the presence of $1 \mathrm{~mm}$ nicotine until photophobic responses could no longer be evoked. For this purpose $500 \mathrm{ml}$ nicotine-containing growth medium was inoculated with $1 \mathrm{ml}$ suspension of bacteria. With bacteria grown under these conditions this procedure was repeated every 5 to 6 days, altogether usually 5 times. Bacteria treated in this way, which will be referred to as "nicotine cells", were used directly from the culture and without further dilution tested immediately after the transfer to the experimental chamber.

\section{Chemicals}

Retinal isomers (all-trans and 13-cis) were obtained from Fluka and purified by high pressure liquid chromatography. 11-cis retinal was a gift of Dr. W. Sperling. L-nicotine and riboflavin were purchased from Fluka and Merck, respectively.

\section{Reconstitution of photoresponses}

Restoration of photosystems after nicotine treatment was examined either after removal of nicotine or after addition of different chromophores. In the first case nicotine cells were harvested by $1.5 \mathrm{~min}$ centrifugation at $10,000 \times g$ (Eppendorf type 5412), washed and resuspended in nicotine-free culture medium. Thereafter bacteria were stored at $38{ }^{\circ} \mathrm{C}$ in the dark until use. Artificial regeneration of photopigments was studied as follows: Ethanolic solution of different retinal isomers and/or carotenoids or riboflavin, respectively, was added to a suspension of nicotine cells in nicotine-containing culture medium at 32 or $38^{\circ} \mathrm{C}$ in the dark. Samples used for determination of action spectra were incubated for at least $12 \mathrm{~h}$ under these conditions.

If not otherwise stated the retinal concentration in the samples was $1.8 \times 10^{-6} \mathrm{M}$. Riboflavin in aqueous and ethanolic solution was used in a similar final concentration. Carotenoids were extracted from $H$. h., strain $\mathrm{R}_{1}$, with methanol/acetone $(1 / 1, \mathrm{v} / \mathrm{v})$ after the purple membrane (PM) had been separated and discarded, and added in a 2- to 3-fold excess (as compared to the content of $\mathrm{R}_{1}$ ) to the sample. The ethanol concentration in all samples tested was usually $0.1 \%$, but never exceeded $0.5 \%$.

\section{Observation and measurements}

Bacteria were observed in a small quartz chamber of about $4 \times 6 \mathrm{~mm}$ width and $0.2 \mathrm{~mm}$ depth covered by a quartz slide under a phase contrast microscope (Leitz, Ortholux, objective: Zeiss, Neofluar $63 \times$ or Ultrafluar $100 \times$, respectively). Background illumination of $\lambda>645 \mathrm{~nm}$ which is not absorbed by retinal was applied through the condensor. Monochromatic stimulating light (half width $\leqq 10 \mathrm{~nm}$ ), variable with respect to wavelength and intensity could be generated either by a xenon lamp (Hanovia, $150 \mathrm{~W}$ ) in connection with a high intensity grating monochromator (Bausch and Lomb, UV/visible range) or a high pressure mercury lamp (Osram, HBO 200) connected with a quartz prism monochromator (Zeiss, M 4 QIII). Light stimuli were applied by an incident light illuminator through the objective lens of the microscope and controlled by an electronic shutter. Usually the stimulus was kept on until the response occurred. In order to measure sensitivity of bacteria towards light, single cells were observed and the latency, the time elapsed between onset of stimulus and the first observable response (stop response), was determined by a stop watch. Either the latency measured upon a constant stimulus or the stimulus intensity necessary to elicit a standard response (constant latency) was used as a measure of sensitivity. Light intensity was measured by means of a light detector (UDT 40) connected with a calibrated pin $10 \mathrm{UV}$. Each measurement was repeated several times with different specimens (see figure legends).

In the reconstitution experiments every sample or at least area of the sample was exposed only once to a light stimulus to avoid photoisomerization of retinal prior to the measurement.

Temperature of the preparation was kept constant $\left( \pm 0.2^{\circ} \mathrm{C}\right.$ ) by means of a thermostatic microscope stage equipped with Peltier elements. Kinetic measurements and determination of action spectra were performed at $32{ }^{\circ} \mathrm{C}$.

Absorption spectra were measured either by a Cary 17 spectrophotometer or a Cary 118 with scattered transmission accessory.

\section{Results}

\section{Effect of nicotine on photophobic responses}

Nicotine is known to inhibit retinal synthesis by blocking cyclization of lycopene to $\beta$-carotene [7]. 


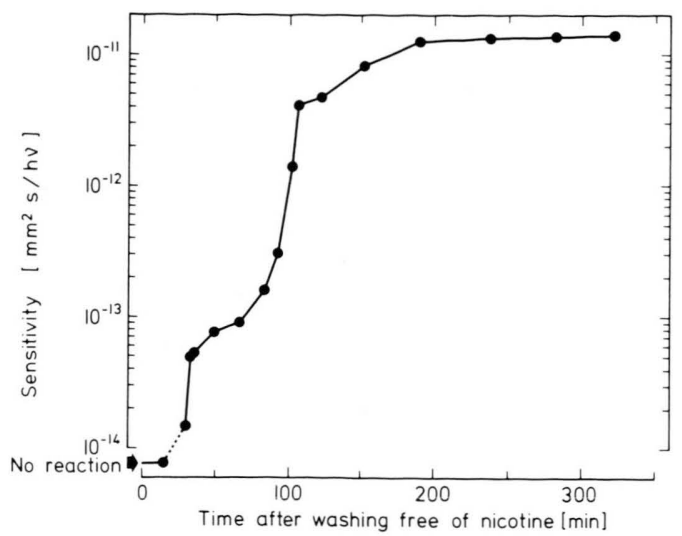

Fig. 1. Recovery of PS 370 in nicotine-grown bacteria after removal of the alkaloid. Ordinate: sensitivity given as the reciprocal of light intensity which evokes a standard response. Each data point was determined after the stimulus intensity had been readjusted several times until at least 19 out of 20 specimens examined showed the response within a given latency of 1.9 to $2.0 \mathrm{~s}$. Stimulating light, $\lambda=370$ $\mathrm{nm}$. Temperature, $38^{\circ} \mathrm{C}$. Sensitivity of $\mathrm{R}_{1} \mathrm{~L}_{3}$ cells before nicotine treatment was $8.3 \times 10^{-12} \mathrm{~mm}^{2} \mathrm{~s} / \mathrm{h} v$.

Consequently halobacteria grown in the presence of nicotine did not form PM which contains BR as the only protein.

Examination of light-dependent behavioral responses revealed that both photosystems became inactive after nicotine treatment. However, normal movement, swimming rate, and the ability to carry out spontaneous reversals were not influenced. Photophobic responses could be evoked neither by decreasing the light intensity at $565 \mathrm{~nm}$ nor by a sudden illumination with UV-light of 280 or $370 \mathrm{~nm}$, respectively. Even stimuli which were about 1000 fold as strong as normally required were found to be ineffective.

The inhibitory effect of nicotine was completely reversible in the case of PS 370 (Fig. 1). After removal of nicotine the photophobic response reappeared. The sensitivity of PS 370 was monitored at $370 \mathrm{~nm}$, and the first response could be detected $30 \mathrm{~min}$ after the washing procedure. Maximum sensitivity was restored after about $200 \mathrm{~min}$ and was as large as before nicotine treatment and as in untreated $R_{1}$ cells (Fig. 4).

\section{Reconstitution of photosystems after nicotine treat- ment}

In a series of experiments different isomers of retinal were added to suspensions of $H$. $h$., strain $\mathrm{R}_{1} \mathrm{~L}_{3}$,

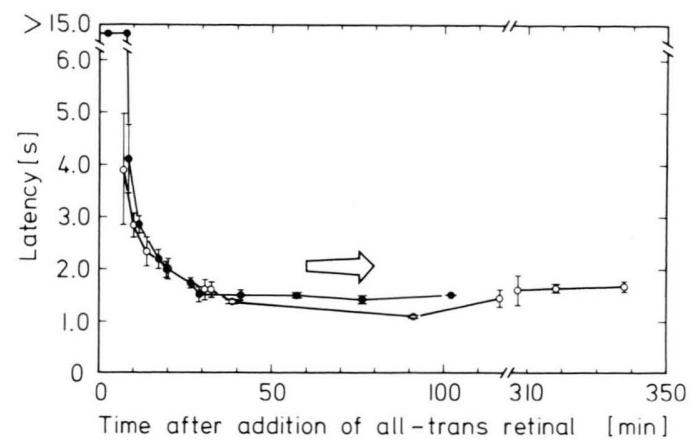

Fig. 2. Reconstitution of PS 370 after addition of all-trans retinal (final concentration $1.8 \times 10^{-6} \mathrm{M}$ ) to nicotine cells. Ordinate: latency between onset of stimulus $\left(4.7 \times 10^{11}\right.$ $\left.\mathrm{h} v \cdot \mathrm{mm}^{-2} \cdot \mathrm{s}^{-1}, \lambda=370 \mathrm{~nm}\right)$ and stop response of bacteria. Different symbols belong to two series of experiments. Data points and vertical bars represent mean \pm SD of 4 to 14 measurements. Arrow depicts reappearance and final latency of PS $565\left(1.5 \times 10^{13} \mathrm{~h} v \cdot \mathrm{mm}^{-2} \cdot \mathrm{s}^{-1}, \lambda=565 \mathrm{~nm}\right)$. Temperature, $32^{\circ} \mathrm{C}$.

after the photobehavior had been blocked by nicotine treatment as described above. To simplify the procedure recovery of PS 370 occurring under these conditions was measured by monitoring the decrease of latency of the photophobic response elicited by a constant light stimulus of $\lambda=370 \mathrm{~nm}$.

Addition of all-trans retinal completely restored PS 370. After a delay of less than $10 \mathrm{~min}$ a fast increase in sensitivity (decrease in latency) occurred which levelled off after 30 to $40 \mathrm{~min}$ (Fig. 2). Sensitivity of PS 565 could first be detected 60 to $80 \mathrm{~min}$ following retinal incubation (depicted by the arrow in Fig. 2). Its final sensitivity, however, was only about one tenth of the value measured in control experiments, i.e. $0.6 \times 10^{-13} \mathrm{~mm}^{2} \mathrm{~s} / \mathrm{h} v$ instead of $5 \times 10^{-13} \mathrm{~mm}^{2} \mathrm{~s} / \mathrm{h} v$ at a given latency of $2.0 \mathrm{~s}$.

Varying the concentration of all-trans retinal resulted in a change of the time elapsed between incubation and the reappearance of PS 370. A response with a latency of $3 \mathrm{~s}$ was observed about 6,12 , and $45 \mathrm{~min}$ after incubation with $9.0 \times 10^{-6}, 1.8 \times 10^{-6}$, and $3.6 \times 10^{-7} \mathrm{M}$, respectively, all-trans retinal. In the latter case the original sensitivity was not completely restored even after $150 \mathrm{~min}$.

A similar increase of the lag period was observed if 13-cis retinal or 11-cis retinal was used instead of all-trans retinal. At equal concentrations of these isomers and constant stimulus strength a response with a 


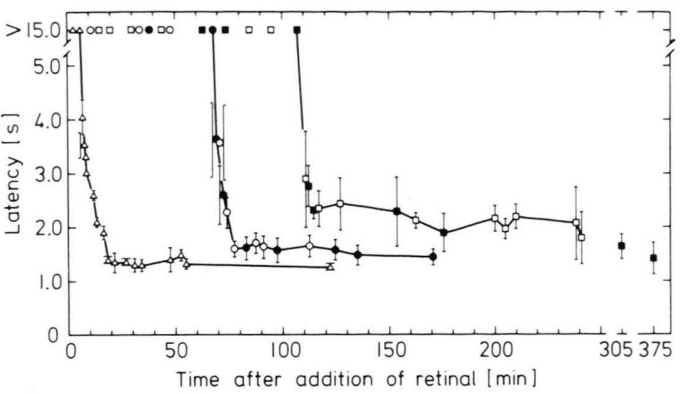

Fig. 3. Reconstitution of PS 370 after addition of all-trans $(\triangle)$, 13-cis $(\bigcirc, \boldsymbol{O})$, and 11-cis retinal $(\square, \mathbf{0})$, respectively (final concentration $1.8 \times 10^{-6} \mathrm{M}$ ). Open and solid symbols represent different series $(n=4-12)$. Ordinate as in Fig. 2. Stimulating light, $8 \times 10^{11} \mathrm{~h} v \cdot \mathrm{mm}^{-2} \cdot \mathrm{s}^{-1}, \lambda=370 \mathrm{~nm}$. Temperature, $38^{\circ} \mathrm{C}$.

latency of $3 \mathrm{~s}$ could be evoked after about 70 or $110 \mathrm{~min}$, respectively, instead of $10 \mathrm{~min}$ in the case of all-trans retinal (Fig. 3). All reconstitution experiments were successful in the presence of $1 \mathrm{~mm}$ nicotine (besides retinal).

Addition of riboflavin and of carotenoids extracted from $H$. $h$., strain $\mathrm{R}_{1}$, to nicotine-treated cells did not restore either PS 370 or PS 565: No photophobic responses could be evoked under these conditions.

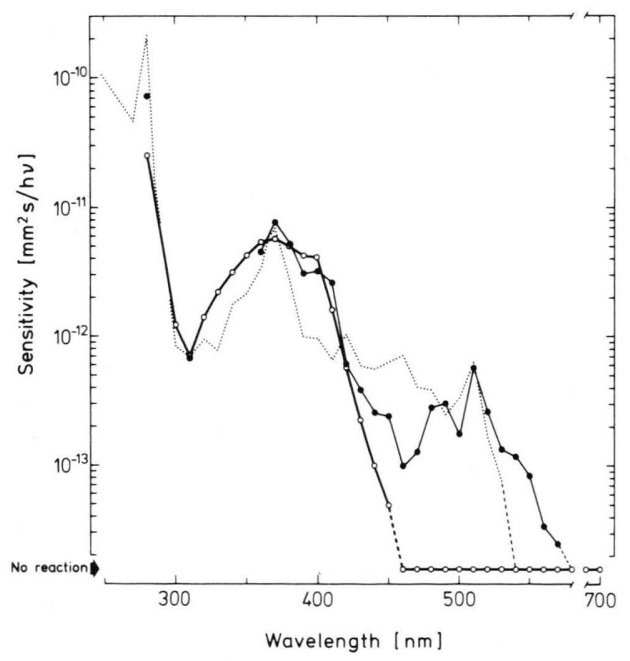

Fig. 4. Action spectra of step-up photophobic response (PS 370) obtained from strain $\mathrm{R}_{1} \mathrm{~L}_{3}$ nicotine cells incubated with all-trans retinal $(O)$ and incubated with all-trans retinal and carotenoids (-). For comparison action spectrum obtained from carotenoid-rich $\mathrm{R}_{1}$ cells (dotted line, from Ref. [3]). Ordinate as in Fig. 1. Background illumination, $\lambda>645 \mathrm{~nm}$. Data points were determined as described in the legend to Fig. 1.

\section{Action spectra}

The sensitivity at $370 \mathrm{~nm}$ of $H$. h., mutant strain $\mathrm{R}_{1} \mathrm{~L}_{3}$, was almost unchanged as compared to that of $\mathrm{R}_{1}$. However, in contrast to the latter no step-up photophobic response could be elicited at wavelengths beyond $450 \mathrm{~nm}$.

The action spectrum obtained from nicotine cells, strain $R_{1} L_{3}$, after reconstitution of PS 370 is given in Fig. 4 (open circles) as compared to the action spectrum of the untreated strain $\mathrm{R}_{1}$ (dotted curve). It shows a marked band with a maximum sensitivity at $370 \mathrm{~nm}$, as in strain $\mathrm{R}_{1}$, but no response beyond $450 \mathrm{~nm}$. Moreover, the 370 band appears much broader than in the case of $R_{1}$ and sensitivity at $280 \mathrm{~nm}$ is significantly reduced.

The light intensity required to elicit a standard response (latency $2 \mathrm{~s}$ ) at $370 \mathrm{~nm}$ was $1.8 \pm 0.2 \times 10^{11}$ $\mathrm{h} v \mathrm{~mm}^{-2} \mathrm{~s}^{-1} \quad(\mathrm{SD}, n=5)$ in reconstituted nicotine cells as compared to $1.5 \pm 0.2 \times 10^{11} \mathrm{~h} v \mathrm{~mm}^{-2} \mathrm{~s}^{-1}$ $(n=7)$ in strain $\mathrm{R}_{1}$.

\section{Effect of carotenoids}

Halobacteria, strain $R_{1} L_{3}$, with reconstituted PS 370 as described above were incubated with carotenoids from strain $\mathrm{R}_{1}$. As a result of this treatment bacteria were markedly sensitized against light of wavelengths between 450 and $570 \mathrm{~nm}$. The action spectrum obtained under these conditions (Fig. 4, solid circles) resembles fairly well that of $\mathrm{R}_{1}$, although some distinct deviations are obvious around $450 \mathrm{~nm}$ and beyond $550 \mathrm{~nm}$. Sensitivity at $280 \mathrm{~nm}$ is somewhat enhanced as compared to reconstituted $\mathrm{R}_{1} \mathrm{~L}_{3}$ without carotenoid treatment but not as high as in $\mathrm{R}_{1}$.

\section{Discussion}

\section{Receptor pigment of PS 370}

The results reported here indicate that the active pigment in PS 370 of H.h. is a UV-absorbing retinal protein complex. Moreover, they support our assumption [3] that the step-down photophobic response (PS 565) is mediated by BR.

Inhibition of PS 370 by nicotine which is known to block retinal synthesis at the stage of $\beta$-carotene formation from lycopene [7] and the ability of retinal to restore this PS point towards a retinylidene protein as the active receptor molecule. The alternative explanation that some step of signal transmission follow- 
ing photoreception by PS 370 requires BR or retinal seems unlikely though this possibility cannot be ruled out with certainty. The action spectrum obtained from retinal-reconstituted $\mathrm{R}_{1} \mathrm{~L}_{3}$ cells (Fig. 4) excludes a flavin protein which has been suggested as the only alternative candidate for the primary photoreceptor of PS 370 [3]. Moreover, no reasonable mechanism is conceivable by which retinal or BR may function in the following process of signal transmission. The fact that the photobehavior can be restored in the presence of nicotine excludes the possibility that the alkaloid itself might block certain steps of signal transmission between stimulus reception and flagellar activation.

Although it seems evident that a retinal protein complex is responsible for sensory transduction of PS 370 the molecular structure of the pigment remains obscure. We were so far unable to detect the pigment spectroscopically or biochemically. However, it is not an uncommon feature of sensory pigments of microorganisms to be present only in very small amounts making it easier to characterize them by means of action spectra than to isolate them.

The action spectrum obtained from bacteria whose PS 370 was reconstituted by retinal (Fig. 4, open circles) matches fairly well the absorption spectrum of a retinylidene protein which can be obtained in vitro after treatment of $\mathrm{PM}$ with $10 \mathrm{mM} \mathrm{CTAB} *$ at alkaline pH [1] (Fig. 5, solid curve). In this complex those non-covalent interactions between chromophore and protein moiety which are thought to cause the bathochromic shift of the absorption spectrum of $\mathrm{BR}$ and visual pigments are obviously absent. The pronounced band at $370 \mathrm{~nm}$ in the action spectrum of PS 370 is probably due to the absorption by the unprotonated Schiff-base linkage.

In nicotine-treated cells no behavioral response could be induced at $280 \mathrm{~nm}$ even at strongly increased light intensity. This observation indicates that a protein by itself cannot trigger the response. Retinal is obviously necessary for sensory transduction and only that energy which is absorbed by a specific protein coupled to the chromophore contributes to excitation.

Reappearance of photoresponses $30 \mathrm{~min}$ after washing the cells free of nicotine (Fig. 1) indicates a fast resumption of retinal synthesis. In the reconstitution experiments all-trans retinal was most effec-

* Cetyltrimethyl-ammoniumbromide, a detergent.

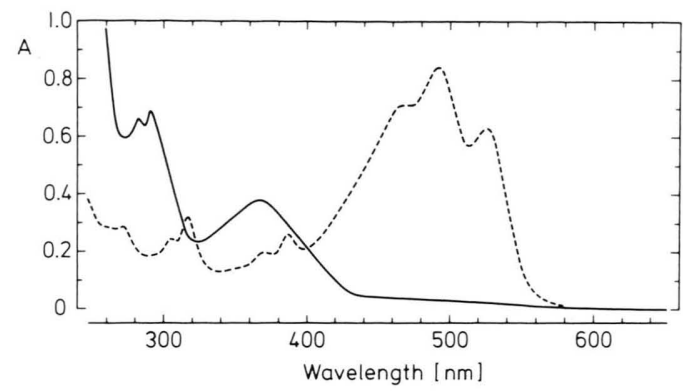

Fig. 5. Absorption spectra of a retinylidene protein obtained after treatment of $\mathrm{PM}$ with $10 \mathrm{mM}$ CTAB at $\mathrm{pH} \approx 8.5$ (solid curve) and of carotenoids extracted from $H$. $h$., strain $\mathrm{R}_{1}$ in ethanol (dashed curve).

tive in restoring PS 370 . We conclude from this that the apoprotein preferentially or solely combines with this isomer and that catalytic isomerization of 13-cis and 11-cis retinal to the trans configuration may take place prior to reconstitution as indicated by the lag time observed in the case of cis isomers (Fig. 3). Photoisomerization by background or stimulating light can be excluded (see Materials and Methods) as well as noncatalytic dark-isomerization, which is negligible at temperatures below $60^{\circ} \mathrm{C}$.

\section{Relation to bacteriorhodopsin}

In the growing culture of $H$. h., strain $\mathrm{R}_{1}$, PS 370 appears about $10 \mathrm{~h}$ earlier than PS 565. It shows maximal sensitivity immediately, whereas PS 565 develops more slowly paralleling the formation of PM [3]. Also in the reconstitution experiments the reappearance of PS 370 preceded that of PS 565 (Fig. 2), which attained only about one tenth of its original sensitivity. The latter is in agreement with the finding that $\mathrm{BO}$ inhibits its own synthesis [8]. Therefore, in nicotine cells the concentration of regenerable $\mathrm{BO}$ is much smaller than the concentration of $\mathrm{BR}$ in untreated cells. The sequence of appearance of photosystems might be a hint that the pigment of PS 370 represents a precursor of BR. This would imply that the protein part of both pigments is identical or principally identical. But also different pathways of biosynthesis are possible.

The result that PS 370 can be fully restored by alltrans retinal although a much smaller concentration of regenerable $\mathrm{BO}$ was found in nicotine cells as compared to the amount of BR in untreated halobacteria [8] argues against an identical protein of both retinal protein complexes. 
The rate of sensitivity increase of retinal-reconstituted PS 370 is comparable to the formation rate of $\mathrm{BR}$ from $\mathrm{BO}$ and all-trans retinal in vitro. However, reappearance of the first inducible step-down photophobic response, mediated by PS 565, is slower than regeneration of BR [9], but faster than PM assembly [8]. The latter fact might indicate that a hexagonal crystalline lattice of $\mathrm{BR}$ is not a prerequisite of PS 565.

\section{Role of carotenoids}

The mutant strain $\mathrm{R}_{1} \mathrm{~L}_{3}$ of $H$. $h$. used in our experiments contains a considerably smaller amount of carotenoids (mainly $\alpha$-bacterioruberin) as compared to strain $R_{1}$. The missing sensitivity around $500 \mathrm{~nm}$ of $R_{1} L_{3}$ cells points to an accessory function of carotenoids in PS 370. Another mutant, $\mathrm{R}_{1} \mathrm{M}_{1}$, which lacks carotenoids too, also does not show the step-up photoresponse at wavelengths beyond $460 \mathrm{~nm}$ [10].

As demonstrated above (Fig. 4, solid circles) carotenoids extracted from $H$. h., strain $\mathrm{R}_{1}$, (Fig. 5, dashed curve) cause a distinct increase in sensitivity to light of wavelengths between 420 and $570 \mathrm{~nm}$ if added to a suspension of bacteria after PS 370 has been restored with retinal. This result supports the conclusion that carotenoids, at least in part, contribute to PS 370 as accessory pigments provided that the retinylidene protein is present. Carotenoids by themselves cannot trigger the behavioral response, but transfer the absorbed energy to the retinal protein complex. This energy transfer from a compound of longer wavelength absorption towards a compound of shorter wavelength absorption may result from a triplet-triplet or exciton interaction. With regard to the relative concentrations of both substances in the living bacterium the efficiency of energy migration seems to be low.

Qualitatively, the absorption spectra of the in vitro modified BR (retinylidene protein) and of extracted carotenoids (Fig. 5) agree with the action spectrum of PS 370 of strain $\mathrm{R}_{1}$ (Fig. 4, dotted line). The slight differences in the position of maxima around $500 \mathrm{~nm}$ are in accordance with the spectral blue shift of carotenoids observable in ethanolic solution. Furthermore, selective loss of membrane-bound carotenoids by the extraction procedure applied can explain the differences between action spectra of $R_{1}$ and reconstituted cells (Fig. 4, solid circles). Absorption spectra of nicotine cells reveal that also synthesis of carotenoids absorbing beyond $380 \mathrm{~nm}$ is inhibited under these conditions. As can be seen in Fig. 4 the band at $370 \mathrm{~nm}$ in the action spectrum of $\mathrm{R}_{1}$ and carotenoid-reconstituted cells of $\mathrm{R}_{1} \mathrm{~L}_{3}$ is sharper as compared with that of cells solely reconstituted with retinal. One possible explanation is that part of the carotenoids act as shielding pigments in this spectral region. However, at present we have no direct experimental proof for this assumption.

Collaboration of a retinal protein complex and carotenoids in photoreceptor function is not a unique feature. The majority of ommatidia of the fly compound eye contains, besides rhodopsin, a photostable dichroic pigment, possibly a carotene, embedded in the membrane of the central rhabdomere. It is suggested that this carotene acts as antenna pigment transferring energy to the UV-sensitive visual pigment [11].

\section{Localization of PS 370}

The PM contains BR as the only protein embedded in the lipid matrix of the cell membrane of $\mathrm{Ha}$ lobacterium [1, 12]. Neither carotenoids nor a UVabsorbing retinylidene protein have been detected in this membrane fraction. With respect to its sensory function PS 370 is most likely also an integral part of the cytoplasmic membrane. We therefore conclude that the active retinal protein complex together with accessory carotenoids, in a special molecular arrangement, are located in membrane areas not occupied by the PM. With respect to the high content of carotenoids in the so-called red membrane [13] we suppose that PS 370 is part of this membrane fraction.

\section{Sensory transduction}

We have so far no direct experimental proof how excitation of the photoreceptor molecule might be coupled to flagellar activity in $H$. $h$. The similarity of behavioral responses elicited by both photosystems suggests a similar mechanism at least of late steps of signal transmission. A sudden decrease of membrane potential, in different ways caused by the photosystems, could well serve as a common signal which triggers flagellar activation [14]. Membrane depolarization is also thought to be involved in behavioral responses of bacteria towards chemical stimuli [15].

In the case of PS 565 depolarization could result from a decrease in electrogenic proton pumping activity of BR after a sudden decrease in light intensity. A similar drop in potential across the membrane 
may be generated by PS 370 if the retinylidene protein acts as a membrane pore or mediates the opening of a pore for a certain ion species $[14,16]$. In contrast to PS 565 the sensitivity of PS 370 was found to be independent of the $\mathrm{pH}$ in the medium (Dencher and Janković, unpublished results). This may indicate that in the latter case protons are not directly involved in the transduction mechanism. Increasing the external $\mathrm{K}^{+}$concentration so as to depolarize the membrane [17] inhibits both photosystems equally well (Hildebrand and Janković, unpublished results).

While the predominant function of PS 565 is obviously photocoupling, i. e. energy conversion, PS 370 acts only as sensory transducer. However, the biological relevance of PS 370 is not clear. It may, together with PS 565, enable the organism to find optimal places for utilization of light energy and to stay

[1] D. Oesterhelt and W. Stoeckenius, Nature New Biol. 233, 149 - 152 (1971).

[2] D. Oesterhelt and W. Stoeckenius, Proc. Nat. Acad. Sci. USA 70, 2853 - 2857 (1973).

[3] E. Hildebrand and N. Dencher, Nature 257, 46-48 (1975).

[4] B. Diehn, M. Feinleib, W. Haupt, E. Hildebrand, F. Lenci, and W. Nultsch, Photochem. Photobiol. 26, 559 - 560 (1977).

[5] D. Oesterhelt and B. Hess, Eur. J. Biochem. 37, 316 - 326 (1973).

[6] P.-J. Bauer, N. A. Dencher, and M. P. Heyn, Biophys. Struct. Mechanism 2, $79-92$ (1976).

[7] C. D. Howes and P. P. Batra, Biochim. Biophys. Acta 222, $174-179$ (1970).

[8] M. Sumper and G. Herrmann, FEBS Letters 69, $149-152$ (1976).

[9] M. E. Keen and N. A. Dencher, EMBO Workshop on Transduction Mechanism of Photoreceptors, Oct. 4-8 (1976), Jülich/Germany, abstract p. 64. there. The aqueous environment of $H$. $h$. may act as a light filter due to selective absorption by pigmentcontaining microorganisms. This determines the spectral composition of sunlight depending on the depth of water. By means of both photosystems the organisms possibly accumulate in a certain layer which corresponds to light conditions favorable for their survival [16]. PS 370 might prevent the cells from damaging irradiation [18] or may represent only an evolutionary remnant [14].

\section{Acknowledgements}

We thank Dr. Lily Jan for a gift of the mutant strain $R_{1} L_{3}$. We are grateful to Dr. Angelika Schimz for critically reading the manuscript. This work was supported by Deutsche Forschungsgemeinschaft (SFB 160).

[10] W. Nultsch and M. Häder, Ber. Dtsch. Bot. Ges. 91, $441-453(1978)$.

[11] K. Kirschfeld and N. Franceschini, Biophys. Struct. Mechanism 3, 191 - 194 (1977).

[12] R. Henderson, Ann. Rev. Biophys. Bioeng. 6, 87 - 109 (1977).

[13] M. Sumper, H. Reitmeier, and D. Oesterhelt, Angew. Chem. Int. Ed. Engl. 15, 187 - 194 (1976).

[14] E. Hildebrand, Taxis and Behavior (ed. G. L. Hazelbauer), pp. 35-73, Chapman and Hall, London 1978.

[15] J. B. Miller and D. E. Jr. Koshland, Proc. Nat. Acad. Sci. USA 74, 4752 - 4756 (1977).

[16] N. A. Dencher, Energetics and Structure of Halophilic Microorganisms, (eds. S. R. Caplan and M. Ginzburg), pp. $67-88$, Elsevier/North Holland, Amsterdam 1978.

[17] G. Wagner, R. Hartmann, and D. Oesterhelt, Eur. J. Biochem. 89, 169 - 179 (1978).

[18] J. D. Dundas and H. Larsen, Arch. Microbiol. 44, $233-239$ (1962). 